\title{
Gert Otto und der Weg der Praktischen Theologie zur Religion in der Gesellschaft
}

\author{
Wilhelm Gräb
}

\section{Anstöße}

Als Gert Otto 1966 eine neue praktisch-theologische Zeitschrift, die Theologia Practica, initiierte, stand diese Initiative bereits im Zeichen einer neuen Konzeption der Praktischen Theologie, die inzwischen für viele Praktische Theologen und Theologinnen maßgeblich geworden ist. Gert Ottos Vorwort ${ }^{1}$ zum ersten Heft der neuen Zeitschrift explizierte das Programm und intonierte zugleich die Melodie, auf die dann seine 20 Jahre später erschienene Gesamtdarstellung der Praktischen Theologie ${ }^{2}$ gestimmt sein sollte: Praktische Theologie ist kritische Theorie religiöser Praxis in der Gesellschaft. Mit dieser These vollzog Otto eine scharfe Abwendung von der bis dahin dominanten Ausrichtung der Praktischen Theologie auf die "Verkündigung der Kirche" und die traditionellen Aufgabenfelder pastoraler Praxis.

Gert Otto stellte sodann das "Verhältnis von Theorie und Praxis" neu zur Debatte, indem er der Theorie einerseits zutraute, der Praxis kritische Impulse zur Veränderung zu vermitteln, er die Praxis andererseits aber auch der Theorie immer schon vorausgesetzt wissen wollte. Das Verständnis der Praktischen Theologie als einer kritischen Theorie religiöser Praxis in der Gesellschaft implizierte eine doppelte Distanznahme, zum einen vom dogmatischen Kirchenbegriff und zum anderen vom Typus der Anwendungswissenschaft.

Die Praktische Theologie sollte sich an die empirische Wirklichkeit der Kirche gewiesen sehen, statt an ekklesiologische Postulate. Ebenso sollte sie die kirchlichen Praxisvollzüge des Predigens, Unterrichts und des seelsorgerlichen Gesprächs im Kontakt mit den Erfahrungs- und Humanwissenschaften, also z. B. der Rhetorik, Didaktik und Gesprächstherapie reflektieren, statt „eine ,bloße' Anwendung exegetischer Erkenntnisse in Predigt, Unterricht und Seelsorge" zu betreiben. ${ }^{3}$ Die Praktische Theologie, so Gert Otto schon in den 60 er Jahren des vergangenen Jahrhunderts, ist eine eigenständige theologische Disziplin. Sie ist weder der Dogmatik, noch den biblischen Fächern als bloße Methoden- bzw. Anwendungsdisziplin nachgeordnet. Ihr Gegenstand, den sie zu erkennen und auf die in ihm liegenden Entwicklungsmöglichkeiten hin kritisch zu reflektieren hat, ist die empirische Wirklichkeit von Kirche und Religion im Kontext der Gesellschaft.

Nachdem Gert 0tto entscheidende Anstöße in dieser Richtung gegeben hatte, er sie schließlich Mitte der 80er Jahre in seiner zweibändigen „Praktischen Theologie" zusammenfasste, führte dies die Praktische Theologie Schritt für

1 Gert Otto: Zur Einführung, in: Theologia Practica 1 (1966), 1-3.

2 Gert Otto: Praktische Theologie, Bd.1, Grundlegung, München 1986; Praktische Theologie, Bd. 2, Handlungsfelder, München 1988.

3 Otto 1966, 1 (Anm. 1). 
Schritt zur Auflösung des durch die Wort-Gottes-Theologie errichteten Verkündigungsparadigmas. Dieses hatte ja doch die Menschen in Kirche und Gemeinde lediglich als Adressaten kerygmatischer Ansprüche in den Blick kommen lassen. Statt dessen wirkte Gert Otto erheblich daran mit, die Eigenständigkeit der Praktischen Theologie und ihren spezifischen Beitrag zum Ganzen der Theologie darin zu erkennen, dass sie nach der Wirklichkeit der Kirche und der Christen in ihr fragt, nach der Wirklichkeit der Predigt, des Unterrichts, der Seelsorge, statt immer nur in normativem Anspruchsdenken zu verharren. Sie analysiert die empirische Kirche, indem sie diese nicht im Gegenüber zur Gesellschaft sieht, sondern als einen Teil von ihr. Sie macht sodann vor allem auch zur Beschreibung der komplexen Interdependenzverhältnisse von Kirche und Gesellschaft vom Religionsbegriff wieder einen unbefangenen Gebrauch.

Inzwischen ist uns dies geläufig. Aber als Gert 0tto Mitte der 70er Jahre diesen Vorschlag machte, die Praktische Theologie "als kritische Theorie religiös vermittelter Praxis in der Gesellschaft ${ }^{\prime 4}$ zu betreiben, sie aus ihrer dogmatisch-ekklesiologischen Engführung zu befreien und für die erfahrungswissenschaftlichen Einsichten in die Wirklichkeit der Kirche im Kontext von Religion und Gesellschaft zu öffnen, war dies ein gewaltiger Umbruch, oder, so könnte man auch sagen, die Rückkehr zu ihren Anfängen.

Bereits um 1900 war von den liberalen Theologen Paul Drews und Friedrich Niebergall betont worden, dass die empirische Wirklichkeit der Religion in Kirche und Gesellschaft sowie im Leben des einzelnen die eigentliche, spezifisch praktisch-theologische Theorieaufgabe darstelle. Ebenso hatte bereits Friedrich Schleiermacher, der Begründer der Praktischen Theologie als einer eigenständigen wissenschaftlich-theologischen Disziplin, ihr ins Stammbuch geschrieben, dass sie die "Theorie der Praxis" ${ }^{\prime 5}$ sei. Als Theorie der Praxis weiß die Praktische Theologie sich in Differenz zu dieser selbst. Aus der Differenz zur Praxis wiederum wächst ihr die Kompetenz zu, die Praxis in ihrer empirischen Wirklichkeit wahrzunehmen, sie in der Aufnahme erfahrungs- und humanwissenschaftlicher Erkenntnisse reflexiv zu durchdringen, um so zu einer bewussten und methodisch geleiteten Gestaltung der Praxis zu befähigen. Gert 0tto hat denn auch zu Recht immer wieder darauf hingewiesen, dass er sich mit seinem Verständnis der Praktischen Theologie als Theorie der Praxis im Gefolge Schleiermachers und mit seinem Pochen auf Empirie und dem Kontakt mit den Humanwissenschaften in der Tradition der liberalen Theologen um 1900 wusste.

\section{Die gelebte Religion als Thema der Praktischen Theologie}

Aber es waren durch Gert Ottos Anstöße nicht nur die empirische kirchliche Wirklichkeit und der Theoriestatus für die Praktische Theologie zurückgewonnen. Gert Ottos entscheidende Innovation bestand des Weiteren darin, dass

${ }^{4}$ Vgl. Gert Otto: Praktische Theologie als kritische Theorie religiös vermittelter Praxis. Thesen zum Verständnis einer Formel, in: Theologia Practica, 9 (1974), 105-115.

${ }^{5}$ Friedrich Schleiermacher: Die praktische Theologie nach den Grundsätzen der evangelischen Kirche im Zusammenhange dargestellt, hg. v. Jacob Frerichs, Berlin 1850, 12. 
er mit der Abkoppelung vom dogmatischen Kirchenbegriff die Kirche nicht mehr in den Gegensatz zur Welt bzw. zur Gesellschaft stellte, sondern sie als denjenigen Teil der Gesellschaft exponierte, in dem sich im Wesentlichen die religiöse Kommunikation in der Gesellschaft abspielt. Mit der empirischen Kirche als Teil der Gesellschaft und daher mit den Menschen, die die Kirche bilden, war zugleich die gelebte Religion als Thema der Praktischen Theologie zurückgewonnen. Die bis dahin herrschende Wort-Gottes-Theologie hatte

\section{Die Praxis der Praktischen Theologie ist die gelebte Religion der Menschen.}

schließlich nicht nur das Verkündigungsparadigma für alle Bereiche kirchlicher Praxis bestimmend gemacht, sondern auch die Frage nach der Religion am Ort der Individuen und als Dimension der Gesellschaft für theologisch irrelevant erklärt. Die Frage nach der Religion war allenfalls als anthropologische, psychologische oder soziologische Frage, nicht aber als praktisch-theologische zugelassen. Denn die Praktische Theologie hatte nach der rechten Ausführung des Auftrags zur Verkündigung zu fragen. Dabei kamen die Menschen, die der Kirche zugehören, lediglich als Adressaten ihrer Botschaft, nicht aber als Subjekte, die sie kommunizieren, in den Blick. Nach ihren Motiven und Interessen, auch und gerade den religiösen Motiven und Interessen wurde nicht gefragt, somit auch nicht danach, wie die Menschen in ihren konkreten Lebenssituationen und mit ihren Lebensinteressen an die kirchliche Kommunikation am ehesten angeschlossen werden können. Da unter dem Regime der Wort-Gottes-Theologie der Hörer der Predigt bei zünftiger Auslegung des biblischen Textes in diesem selbst sollte gefunden werden können, stellte die Hermeneutik der Kultur der Gegenwart insgesamt, somit auch die Erforschung der Rezeptionsbedingungen kirchlicher Kommunikation, kein praktisch-theologisches Thema dar.

Demgegenüber hat Gert 0tto zusammen mit Dietrich Rössler, der in das erste Heft von "Theologia Practica" ebenfalls einen entsprechenden Beitrag eingebracht hatte, ${ }^{6}$ energisch den erneuten Aufbruch der Praktischen Theologie in jenes unbekannte Land betrieben, das sie an der Wende zum 20. Jahrhundert schon einmal von Ferne gesehen hatte: die "sozialkulturelle Lebenswelt der christlichen Religion“ (Volker Drehsen). Diese erneute Hinwendung zur empirischen Wirklichkeit der Kirche, zur gelebten Religion am Ort der Individuen und in den Funktionszusammenhängen der Gesellschaft, der damit verbundene Verzicht auf ekklesiologische Deduktionen, die Offenheit für neue kirchliche Herausforderungen im Spannungsfeld von Religion und Gesellschaft charakterisieren schließlich Gert Ottos "Praktische Theologie“ wesentlich. Die Praxis der Praktischen Theologie ist die gelebte Religion der Menschen. Sie gilt es zunächst wahrzunehmen und kritisch zu reflektieren, um die kirchlichen Handlungsvollzüge der Predigt, des Unterrichts, der Seelsorge usw. daraufhin zu befragen, ob sie die Menschen in der Wirklichkeit ihres Lebens und Glaubens zu erreichen, zu stärken und zu orientieren in der Lage sind. Der entscheidende Punkt war eben der, dass Gert Otto diese Praxis der gelebten

\footnotetext{
${ }^{6}$ Vgl. Dietrich Rössler: Das Problem der Homiletik, in: Theologia Practica 1 (1966), 14-28.
} 
Religion wieder zu einer eigenständigen und entscheidenden Theorieaufgabe der Praktischen Theologie gemacht hat, statt sie lediglich die Applikationsfläche für exegetische Einsichten, dogmatische Wesensaussagen und normative Verkündigungsansprüche sein zu lassen.

Der Praxisbegriff war damit natürlich auch sehr weit gefasst. Die Praxis, die die Praktische Theologie zu thematisieren hat, ist letztendlich die Praxis des Lebens überhaupt, freilich unter dem spezifischen Aspekt religiöser Sinn- und Handlungsbezüge. Aber die religiöse Dimension lässt sich in den gesellschaftlichen und individuellen Lebensbezügen natürlich auch nicht so leicht abgrenzen, der Gegenstand der Praktischen Theologie somit nicht so klar festlegen wie dies am Leitfaden der dogmatischen Ekklesiologie und ihrer Wesensaussagen über die Grundvollzüge kirchlichen Lebens möglich ist. Dafür öffnet die Religionsthematik aber den Blick für die Themen der Zeit, die sowohl existenziell wie gesellschaftlich, jedenfalls nicht nur binnenkirchlich relevant sind. Sie werden von Gert 0tto im ersten Abschnitt des 2. Bandes seiner Gesamtdarstellung beschrieben. ${ }^{7}$ Es sind die Fragen nach personaler und religiöser Identität, nach den gesellschaftlichen Wertorientierungen (Civil Religion), nach den Chancen zur Wahrung kultureller Herkunftsbestände im Dynamismus der modernen Gesellschaft, nach dem Beitrag des christlichen Glaubens zu einer Ethik der Humanität.

\section{Die Entkirchlichung der Praktischen Theologie}

Mit der Ausrichtung auf die modernen Herausforderungen individueller und gesellschaftlicher Lebenspraxis, wie sie sich im Horizont der religiösen Sinndimension stellen, konnte Gert Otto die Praktische Theologie über binnenkirchliche Selbstverständlichkeiten hinausführen und zu Überlegungen gelangen, wie die kirchliche Praxis nach außen hin ihre Kommunikation zu verbessern vermag. So hat Gert 0tto insbesondere in seinen homiletischen und religionspädagogischen Überlegungen deutlich gemacht, dass es auf hermeneutische, rhetorische und didaktische Übersetzungsleistungen ankommt, wenn die Vermittlung des christlichen Glaubens auch denjenigen Zeitgenossen gegenüber, die von seiner Wahrheit nicht unbedingt schon überzeugt, aber offen für existenzielle und religiöse Sinnfragen sind, soll gelingen können. Insbesondere die Predigt und der Religionsunterricht sollten dazu fähig sein, kritische, gerade auch kirchen- und religionskritische Zeitgenossen von der Lebensdienlichkeit des christlichen Glaubens überzeugen zu können.

Mit seinem Verständnis von der "Predigt als Rede“ hat Gert Otto für die energische Integration einer hermeneutischen Rhetorik in die Grundfragen der Homiletik gekämpft. ${ }^{8}$ "Predigt als Rede“ geht nicht von feststehenden biblischen oder dogmatischen Wahrheiten aus, sondern versucht, auf dem Wege einer situationsbezogenen und gegenwartssensiblen Auslegung der bib-

7 Otto 1988, 23-62.

8 Vgl. Gert Otto: Predigt als Rede. Über die Wechselwirkungen von Homiletik und Rhetorik, Stuttgart u.a. 1976. 
lischen Texte lebensdienliche Wahrheit, die für Zeitgenossen heute konkret wird, zu finden und gewinnend zur Sprache zu bringen. Mit der gleichen Absicht einer Orientierung der kirchlich-religiösen Praxis nicht an kirchlich-dogmatischen Vorgaben, sondern an den Sinnorientierungsinteressen der Menschen setzte Gert 0tto sich in seiner Religionspädagogik für die Überwindung des konfessionellen Religionsunterrichts und für die Aufhebung der Scheinalternative "Religion oder Ethik?" ein. ${ }^{9}$

In der Homiletik hat Gert 0tto sich weithin durchgesetzt, wird doch die Frage nach der Gestalt der Predigt als einer performativen Rede bzw. eines dramaturgischen Geschehens gerade heute wieder heftig diskutiert. Leider fand Otto mit seinem Plädoyer für einen "didaktisch zureichend reflektierten „EthikUnterricht" als „Religionsunterricht für alle ${ }^{\mu 10}$ kaum Gehör. Dabei hätte man darauf ein tragfähiges Konzept gerade für den Religionsunterricht in den neuen Bundesländern aufbauen können. Weil man in diese Richtung zu denken insbesondere auf Seiten der Kirche nicht ansatzweise bereit war, ist die Chance der Integration der Religionsthematik in den Ethikunterricht vertan worden. So haben wir jetzt z. B. in Berlin einen "Ethikunterricht für alle", aber ohne "Religion".

Mit der Abwendung von der dogmatischen Ekklesiologie stand für Gert 0tto seit dem Start von "Theologia Practica“ auch die traditionelle Einteilung der Praktischen Theologie in Kybernetik, Liturgik, Homiletik, Katechetik, Poimenik

Leider fand Gert Otto mit seinem Plädoyer für einen "Ethikunterricht" als "Religionsunterricht für alle" kaum Gehör. usw. zur Disposition. Im Untertitel des 1. Heftes wurde diese Umstellung dadurch zur Kenntnis gebracht, dass die "Theologia Practica" als "Zeitschrift für Praktische Theologie und Religionspädagogik" firmierte. Die "Religionspädagogik" stellt ja seit ihren Anfängen in der liberalen Theologie um 1900 diejenige praktisch-theologische Disziplin dar, die sich in eine pastoraltheologisch oder kirchentheoretisch verengende Praktische Theologie nicht integrieren lässt. Deshalb sollte die "Theologia Practica" eine Praktische Theologie befördern, die nicht "nur das Praktikable für , die Kirche' oder für ,den Pfarrer' im Blick hat ${ }^{\prime \prime 11}$, sondern vielmehr die Fragen angeht, die über die pastorale Berufspraxis und die konstitutiven Grundvollzüge der Kirche hinaus „am Treffpunkt von Evangelium und Welt" aufbrechen. Mit dieser Öffnung zur "Welt" hin vollzog sich diejenige praktisch-theologische Theorietransformation, die Gert 0tto dann in die konstruktive Orientierung der ganzen Disziplin an den Begriffen der "Gesellschaft" wie auch der "Religion“ aufnahm. 1974 stellte er seine Theorie denn auch unter die schon erwähnte Leitformel: „Praktische Theologie als kritische Theorie religiös vermittelter Praxis in der Gesellschaft. ${ }^{.12}$

Mit dieser Formel Praktischer Theologie ist die Kirche um die Gesellschaft er-

\footnotetext{
9 Vgl. Gert Otto: „Religion“ contra "Ethik“?, München 1986.

10 Otto 1988, 121.

11 Vgl. Otto 1966, 2

12 Vgl. Gert Otto: Praktische Theologie als kritische Theorie religiös vermittelter Praxis. Thesen zum Verständnis einer Formel, in: Theologia Practica, 9 (1974), 105-115.
} 
weitert und die Kirche als derjenige Teil der Gesellschaft begriffen, mit dem es um die religiöse Kommunikation in der Gesellschaft geht. Des Weiteren ist mit dieser Formel an die Stelle einer den kirchlichen Verkündigungsauftrag instrumentalisierenden Praktischen Theologie die kritische Reflexion, das Verstehen und Deuten der gelebten Religion der Menschen getreten. „Religion, Gesellschaft und Kirche“ (der spätere Untertitel von Theologia Practica und Leitgesichtspunkt in 0ttos Gesamtdarstellung der Praktischen Theologie) beschreiben nun diejenigen Kommunikationszusammenhänge, die die Praktische Theologie kritisch zu analysieren und für die sie ihre Theorien zur Verbesserung der religiösen Kommunikation auszubilden hat. Jedes Phänomen gesellschaftlicher Praxis kann, sofern es irgendwie im Zusammenhang mit "Religion“ steht, auch Gegenstand religiöser Kommunikation und damit praktisch-theologischer Theoriearbeit werden.

\section{Die religiösen Individuen als Subjekte der Praxis}

Diese Ausweitung im Praxisbegriff, weit über die Kirche und die pastoralen Grundfunktionen hinaus, hin zur "konkreten Vielfalt von Menschen mit ihren je unterschiedlichen Beziehungen zu Religion und Kirche ${ }^{\mu 13}$, ließ sich mit der überkommenen sektoralen Gliederung der Praktischen Theologie nicht mehr vereinbaren. An die Stelle der Kirche als handelndem Subjekt sind bei Otto die religiösen Individuen getreten, mit ihrer Nähe und Distanz zur Kirche, mit ihrer religiösen Indifferenz und ihrem kirchlichen Engagement. Weil es um die Individuen und ihre Religion, sodann auch um eine Kirche geht, die die Menschen in ihren Lebenssituationen und religiösen Lebensfragen erreicht und anspricht, insistierte 0tto darauf, dass "alle und nicht nur die Kleriker und nicht nur die kerngemeindlichen Christen als Subjekte wahr- und ernstzunehmen" sind. ${ }^{14}$ Die Individuen sind im Blick auf die religiöse Dimension ihres Menschseins von der Praktischen Theologie "wahr- und ernstzunehmen“, aber damit zugleich immer auch im Kontext von Gesellschaft, von Politik und Erziehung, Wirtschaft und Recht, Ehe und Familie, Sozialisation und Bildung, Krieg und Frieden, Umwelt und Ökologie usw.

Diese Themenfelder der Praktischen Theologie lassen sich nicht mehr in ein ekklesiologisches System fügen. Hinzu kommt, dass die Praktische Theologie in Anlehnung an Horkheimers Unterscheidung von traditioneller und kritischer Theorie, die 0tto aufgenommen hatte, kritische Theorie der gelebten Religion sein sollte, die Religion somit nicht nur beschreiben, sondern die bislang verstellten und noch unausgeschöpften Möglichkeiten des Menschen in ihrem religiösen Verhältnis aufdecken sollte. Gert Otto versuchte der Praktischen Theologie zu Recht auch einen ideologiekritischen Zug einzuschreiben mit dem Ziel, dass sie Potenziale der Veränderung in Kirche und Gesellschaft sichtbar macht.

13 Otto 1986, 62.

14 A.a.0., 63. 
Wenn gilt, dass die Praktische Theologie "alle [...] als Subjekte wahr- und ernstzunehmen" hat, dann kann gleichwohl der Anschein entstehen, als habe die Praktische Theologie jedes Maß disziplinärer Beschränkung verloren. Das wäre allerdings ein Missverständnis. Auch Gert 0tto fokussierte seine als kritische Theorie gelebter Religion konzipierte Praktische Theologie weiterhin auf

Die Lebenswelt der christlichen

Religion ist das Terrain, auf dem sich die Praktische Theologie zu bewegen hat.

die kirchlichen Handlungsfelder. Es sollten sich also auch weiterhin die Pfarrer und Pfarrerinnen als die Akteure auf diesen Handlungsfeldern angesprochen sehen. Mit dem Subjektstatus der Individuen war gemeint, dass deren Lebensfragen das Thema der kirchlichen Kommunikation sein sollten, sei es in der Erwachsenenbildung, in der Predigt, im Religionsunterricht, in der Seelsorge. Nie sind die Menschen bloße Adressaten kirchlicher Belehrungs- und Verkündigungsansprüche, sondern sie sind Subjekte in der Aneignung von kirchlich-religiösen Kommunikationsangeboten. In diesem Sinne gilt es nach den spezifischen kirchlichen Herausforderungen zu fragen, die aus den gesellschaftlich notwendigen Transformationsprozessen und aus dem Subjektstatus der Menschen, für die die Kirche als Ort religiöser Kommunikation da ist, erwachsen. Auch wenn dabei die traditionelle sektorale Gliederung der Praktischen Theologie nicht aufrecht zu erhalten ist, die traditionellen Bereiche der kirchlichen Praxis kommen doch alle wieder vor. Predigt und Gottesdienst, die Kasualien, Kindergottesdienst, Religions- und Konfirmandenunterricht und Jugendarbeit, Seelsorge und Psychotherapie im kirchlichen Kontext, die kirchlich verantwortete oder mitverantwortete Bildungs- und Kulturarbeit stecken ein offenes Terrain ab. Entscheidend ist, dass die praktisch-theologische Aufgabe auf allen diesen Handlungsfeldern im Kern darin gesehen wird, die Religion am Ort der sie lebenden Subjekte wahrzunehmen und in ihren ganzen Ambivalenzen und Fraglichkeiten zu verstehen, um sie im Medium der Auslegung überlieferter biblischer Texte und anderer Materialien tiefer über sich zu verständigen und anderen kommunizieren zu können. Die Lebenswelt der christlichen Religion, für die die Kirche wichtig bleibt, die in ihr aber nicht aufgeht, ist das Terrain, auf dem sich die Praktische Theologie zu bewegen hat.

Das ist heute weithin als die zentrale Aufgabe Praktischer Theologie erkannt. Die Religionsthematik festigt ihre herausragende Position. Sie wird jedoch, stärker als dies noch bei Gert 0tto der Fall war, phänomenologisch und hermeneutisch angegangen. Die Weite des Blicks auf die Gesellschaft bleibt aber ebenso wichtig. Inzwischen sind lediglich die Fragen der ästhetischen Kultur, des Lebensstils und der Lebenskunst, der Civil Religion und des interreligiösen Dialogs, der Ökumene vor Ort, Fragen des Verhältnisses von Kirche und Kultur, von populärer Kultur und Religion stärker in den Vordergrund gerückt. Die kulturphänomenologische und -hermeneutische Ausrichtung der Praktischen Theologie hat sich in einer Weise durchgesetzt, die nicht gegen das handlungswissenschaftliche Selbstverständnis der Praktischen Theologie, an dem Gert 0tto orientiert geblieben ist, ausgespielt werden muss. Im Sinne der 
kritischen Theorie gelebter Religion, der Gert Otto den Weg gebahnt hat, wird vielmehr auch heute als praktisch-theologische Aufgabe erkannt, dass es Orientierungen für die kirchliche Praxis zu gewinnen gilt. Sie sollten dazu helfen, die Kirche als einen relevanten Ort lebensdienlicher religiöser Kommunikation in der Gesellschaft zu erhalten.

\section{Von Wandlungen, Variationen und "konzeptionellem Kettenriss" - Gert Otto und die Debatte über Religion in der Schule}

Jürgen Lott

Der Name Gert Otto ist nicht allein mit der Praktischen Theologie, speziell der Homiletik, sondern vor allem auch mit der (schulischen) Religionspädagogik der 2. Hälfte des 20. Jahrhunderts aufs Engste verbunden:

- Religionsunterricht als "hermeneutische Aufgabe“,

- "Kritischer Religionsunterricht“ in einem demokratischen Schulwesen,

- "bikonfessioneller" Religionsunterricht,

- "Religion“ contra "Ethik",

- "Allgemeine Religionskunde - Religionsunterricht für alle",

das sind die wichtigsten Stichworte und Positionen, die Gert Otto in der Religionsdidaktik nach 1950 und bis zu seinem Tod im Februar 2006 vertreten hat. Rainer Lachmann hat bereits vor über 25 Jahren in seiner Bamberger Antrittsvorlesung 0ttos "religionspädagogische Entwicklung" thematisiert, um daran grundsätzlich „Religionspädagogische Wandlungen als wissenschaftliches und ethisches Legitimationsproblem ${ }^{\prime 1} \mathrm{zu}$ untersuchen. In diesem $\mathrm{Zu}$ sammenhang hält er Gert Otto unsachgemäße „Anpassung an den Zeitgeist" vor.

\section{Von der „Evangelischen Unterweisung" zum „hermeneutischen Religionsunterricht" oder - die „Entwicklung" Gert Ottos}

Zunächst zeichnet Lachmann im Verlauf dieser Abhandlung, die ich aufgreife, weil sie beispielhaft für die Bewertung der religionspädagogischen Entwicklung 0ttos durch die universitären religionspädagogischen Fachkollegen stehen kann, anhand von Original-Zitaten Gert Ottos Weg von einem Vertreter

1 Rainer Lachmann: Religionspädagogische Wandlungen als wissenschaftliches und ethisches Legitimationsproblem exemplifiziert an Gert Ottos religionspädagogischer Entwicklung, in: EvErz 33 (1981), Heft 2, 86ff. 